\title{
A Gårding Domain for Quantum Fields
}

\author{
Michael C. ReED \\ Department of Mathematics, Princeton University
}

Received June 25, 1969

\begin{abstract}
In all representations of the canonical commutation relations, there is a common, invariant domain of essential self-adjointness for quantum fields and conjugate momenta.
\end{abstract}

\section{Introduction}

Let $U_{k}(s), V_{k}(t)$ be one-parameter continuous unitary groups on a separable Hilbert space $X$, satisfying the relations:

$$
\begin{aligned}
U_{k}(s) V_{l}(t) & =e^{i s t \delta_{k l} l} V_{l}(t) U_{k}(s), \\
{\left[U_{k}(s), U_{l}(t)\right] } & =0=\left[V_{k}(s), V_{l}(t)\right]
\end{aligned}
$$

for all $k, l=1,2, \ldots$ and $s, t \in R$. Such a structure is called a representation of the Weyl relations. In this paper we prove the following theorem (whose consequences for Quantum Field Theory are discussed in $\S 5$ ).

Theorem 1.1. Let $\left(\left\{U_{k}(s), V_{k}(t)\right\}_{k=1}^{\infty}, X\right)$ be a representation of the Weyl relations; denote by $p_{k}$ the generator of $U_{k}(s)$, by $q_{k}$ the generator of $V_{k}(t)$. Then there exists a Banach space, $\tau$, of sequences of real numbers and a domain $D$, dense in $X$, such that for all $\left\{c_{k}\right\}_{k=1}^{\infty} \in \tau$,

1) $\sum_{k=1}^{\infty} c_{k} q_{k}, \sum_{k=1}^{\infty} c_{k} p_{k}$ are well-defined and essentially self-adjoint on $D$,

2) $\sum_{k=1}^{\infty} c_{k} q_{k} D \subset D, \sum_{k=1}^{\infty} c_{k} p_{k} D \subset D$.

3) If $\left\{c_{k}^{n}\right\}_{k=}^{\infty} \stackrel{\iota}{\rightarrow}\left\{c_{k}\right\}_{k=1}^{\infty}$ and $\varphi \in D$, then

$$
\sum_{k=1}^{\infty} c_{k}^{n} q_{k} \varphi \rightarrow \sum_{k=1}^{\infty} c_{k} q_{k} \varphi \text { and } \sum_{k=1}^{\infty} c_{k}^{n} p_{k} \varphi \rightarrow \sum_{k=1}^{\infty} c_{k} p_{k} \varphi
$$

We remark that if we were concerned with only a finite number of $q_{k}$ and $p_{k}$, the conclusions of the theorem would follow from well-known work of L. Gårding on representations of Lie groups. For the Fock representation the theorem was proven by J. Cook [2]. In our proof we use heavily the classification of all representations achieved by Garding and Wightman [4]; it is briefly described in $\S 2$.

The proof of the theorem is contained in $\S 3$ and $\S 4$. In $\S 3$ we construct a dense set of vectors $D_{1} \subset X$. The construction is done so that for 
$\psi \in D_{1}$, one has precise estimates on the growth of $\left\|\left(p_{k}^{2}+q_{k}^{2}+1\right) \psi\right\|$ as $k \rightarrow \infty$. We then show that $\sum c_{k} \mathrm{q}_{k}, \sum c_{k} p_{k}$ are well-defined symmetric operators on $D_{1}$, if $\left\{c_{k}\right\}_{k=1}^{\infty} \in \tau$, a sequence space depending on the estimates. In $\S 4$ we enlarge $D_{1}$ to a domain $D$ by allowing certain infinite linear combinations of vectors in $D_{1}$. Using the estimate

$$
\left\|\prod_{i=1}^{N} q_{k_{i}} \psi\right\| \leqq 2^{N / 2} N^{N / 2}\left\|\prod_{i=1}^{N}\left(p_{k_{i}}^{2}+q_{k_{i}}^{2}+1\right)^{1 / 2} \psi\right\|
$$

we show that each $\psi \in D$ is an analytic vector for $\sum_{k=1}^{\infty} c_{k} q_{k}$ and $\sum_{k=1}^{\infty} c_{k} p_{k}$, thus proving essential self-adjointness. Invariance and continuity follow trivially from the construction.

Anticipating the notation introduced in $\S 2$ we remark here that the test sequence space $\tau$ depends only on the Gårding-Wightman measure $\mu$, not on the reducibility of the representation or the fibre maps $C(\tilde{n})$. Also, there will be many nuclear spaces and Hilbert spaces (with stronger topologies) contained in $\tau$ so that in the statement of the theorem "Banach" may be replaced by "nuclear," or "Hilbert."

\section{§ 2. The Gårding-Wightman Classification}

Let $I^{\infty}$ denote the set of all sequences $\tilde{n}=\left(n_{1}, n_{2}, \ldots, n_{k}, \ldots\right)$ of nonnegative integers. Let $I_{k j}$ be the set of sequences such that $n_{k}=j$, and denote by $\mathscr{M}$ the smallest $\sigma$-algebra containing $\left\{I_{k j}\right\}_{k, j=1}^{\infty}$. A measure $\mu(\tilde{n})$ on $\left(I^{\infty}, \mathscr{M}\right)$ is called quasi-invariant if $\mu(\tilde{n})$ and $\mu(\tilde{n}+\delta)$ are equivalent whenever $\delta$ is a sequence of integers with only a finite number of non-zero entries. Let $\mu$ be a quasi-invariant measure of mass one and let $v(\tilde{n})$ be a quasi-invariant positive integer-valued function on $I^{\infty}$ and let $H_{\tilde{n}}$ be a Hilbert space of dimension $v(\tilde{n})$. We denote by $L^{2}\left(I^{\infty}, \mu(\tilde{n}), H_{\tilde{n}}\right)$ the direct integral $\int_{I^{\infty}} H_{\tilde{n}} d \mu(\tilde{n})$. We define operators $a_{k}$ and $a_{k}^{*}$ on all vectors $f(\tilde{n}) \in L^{2}\left(I^{\infty}, \mu(\tilde{n}), H_{\hat{n}}\right)$ satisfying

by the formulas

$$
\int_{I^{\infty}} n_{k}\|f(\tilde{n})\|_{\tilde{n}}^{2} d \mu(\tilde{n})<\infty
$$

$$
\begin{aligned}
& \left(a_{k} f\right)(\tilde{n})=\sqrt{n_{k}+1} C_{k}(\tilde{n}) f\left(\tilde{n}+\delta_{k}\right) \sqrt{\frac{d \mu\left(\tilde{n}+\delta_{k}\right)}{d \mu(\tilde{n})}} \\
& \left(a_{k}^{*} f\right)(\tilde{n})=\sqrt{n_{k}} C_{k}^{*}\left(\tilde{n}-\delta_{k}\right) f\left(\tilde{n}-\delta_{k}\right) \sqrt{\frac{d \mu\left(\tilde{n}-\delta_{k}\right)}{d \mu(\tilde{n})}}
\end{aligned}
$$

where $C_{k}(\tilde{n})$ are measurable unitary operators from $H_{\tilde{n}+\delta_{k}}$ to $H_{\tilde{n}}$ satisfying $C_{k}(\tilde{n}) \mathrm{C}_{l}\left(\tilde{n}+\delta_{k}\right)=C_{l}(\tilde{n}) \mathrm{C}_{k}\left(\tilde{n}+\delta_{l}\right)$. In the above $\|f(\tilde{n})\|_{\tilde{n}}$ is the norm of $f(\tilde{n})$ in $H_{\tilde{n}}$ and $\delta_{k}$ denotes the sequence with one in the $k$ th place and zero elsewhere. 
If we define $q_{k}=\frac{i}{\sqrt{2}}\left(a_{k}-a_{k}^{*}\right)$ and $p_{k}=\frac{1}{\sqrt{2}}\left(a_{k}+a_{k}^{*}\right)$, then their closures are self-adjoint and the groups they generate are a representation of the Weyl relations. Furthermore, every representation is unitarily equivalent to one of these; therefore in the proof of the theorem stated in $\S 1$ we may assume that $X=L^{2}\left(I^{\infty}, \mu(\tilde{n}), H_{\tilde{n}}\right)$ and that $q_{k}$ and $p_{k}$ are given by the formulas (2.1).

\section{§ 3. Construction of the Analytic Vectors}

Let $\mu$ be a quasi-invariant Borel measure of mass one on $I^{\infty}$. We proceed to define a family of measurable sets $\left\{I^{l}\right\}_{l=1}^{\infty}$ such that $I^{l} C I^{l+1}$ and $\mu\left(I^{l}\right) \geqq 1-\frac{1}{2^{l}}$. In this construction all sets are obtained by countable processes from sets in $\mathscr{M}$ and are thus measurable. Since $I^{\infty}=\bigcup_{j=1}^{\infty} I_{1 j}$ is a disjoint decomposition, $1=\mu\left(I^{\infty}\right)=\sum_{j=1}^{\infty} \mu\left(I_{1 j}\right)$. Choose an integer $\varrho(1,1)$ such that $\sum_{j>\varrho(1,1)} \mu\left(I_{1 j}\right)<\left(\frac{1}{2}\right)\left(\frac{1}{2}\right)$, and define $J_{(1,1)}=\bigcup_{j=1}^{\varrho(1,1)} I_{1 j}$; note that $\mu\left(J_{(1,1)}\right) \geqq 1-\left(\frac{1}{2}\right)\left(\frac{1}{2}\right)$. Now, $J_{(1,1)}=\bigcup_{j=1}^{\infty}\left(J_{(1,1)} \cap I_{2 j}\right)$; choose $\varrho(1,2) \geqq \varrho(1,1)$ such that $\sum_{j>\varrho(1,2)} \mu\left(J_{(1,1)} \cap I_{2 j}\right) \leqq\left(\frac{1}{2}\right)\left(\frac{1}{2}\right)^{2}$, and define $J_{(1,2)}=\bigcup_{j=1}^{\varrho(1,2)}\left(J_{(1,1)} \cap I_{2 j}\right) ;$ note that $\mu\left(J_{(1,2)}\right) \geqq 1-\left(\frac{1}{2}\right)\left(\frac{1}{2}\right)-\left(\frac{1}{2}\right)\left(\frac{1}{2}\right)^{2}$. We proceed inductively, defining $J_{(1, k)}=\bigcup_{j=1}^{\varrho(1, k)}\left(J_{(1, k-1)} \cap I_{k j}\right)$ where $\varrho(1, k)$ $\geqq \varrho(1, k-1)$ is chosen so that $\sum_{j>\varrho(1, k)} \mu\left(J_{(1, k-1)}^{j=1} \cap I_{k j}\right) \leqq\left(\frac{1}{2}\right)\left(\frac{1}{2}\right)^{k}$.

Thus $\mu\left(J_{(1, k)}\right) \geqq 1-\frac{1}{2}\left(\sum_{i=1}^{k}\left(\frac{1}{2}\right)^{i}\right)$. We now set

$$
I^{1}=\bigcap_{k=1}^{\infty} J_{(1, k)}
$$

and observe that $\mu\left(I^{1}\right)=\lim _{k \rightarrow \infty} \mu\left(J_{(1, k)}\right) \geqq \frac{1}{2}$. We have defined a nondecreasing function on the positive integers, $\varrho(1, \cdot)$.

Having defined $I^{1}$ and $\varrho(1, \cdot)$ we proceed by induction to define $I^{l}$ and $\varrho(l, \cdot)$. We choose $\varrho(l, 1) \geqq \varrho(l-1,1)$ so that $\sum_{j>\varrho(l, 1)}^{\infty} \mu\left(I_{1 j}\right) \leqq\left(\frac{1}{2}\right)^{l}\left(\frac{1}{2}\right)$. Letting $J_{(l, 1)}=\bigcup_{j=1}^{\varrho(l, 1)} I_{1 j}$ we define $J_{(l, k)}$ by induction as $\bigcup_{j=1}^{\varrho(l, k)}\left(J_{(l, k-1)} \cap I_{k j}\right)$ 
where $\varrho(l, k)$ is chosen so that

and

$$
\varrho(l, k) \geqq \varrho(l-1, k), \varrho(l, k) \geqq \varrho(l, k-1)
$$

$$
\sum_{j>\varrho(l, k)}^{\infty} \mu\left(J_{(l, k-1)} \cap I_{k j}\right) \leqq\left(\frac{1}{2}\right)^{l}\left(\frac{1}{2}\right)^{k}
$$

Setting $I^{l}=\bigcap_{k=1}^{\infty} J_{(l, k)}$ we find that

$$
\begin{aligned}
\mu\left(I^{l}\right)=\lim _{k \rightarrow \infty} \mu\left(J_{(l, k)}\right) & \geqq 1-\left(\frac{1}{2}\right)^{l}\left(\sum_{i=1}^{\infty}\left(\frac{1}{2}\right)^{i}\right) \\
& =1-\left(\frac{1}{2}\right)^{l} .
\end{aligned}
$$

The sets $I^{l}$ are increasing and $\mu\left(\bigcup_{l=1} I^{l}\right) \geqq 1-\left(\frac{1}{2}\right)^{l}$ for all $l$, so $\mu\left(\bigcup I^{l}\right)=1$. Each of the functions $\varrho(l, \cdot)$ is non-decreasing and $\varrho(l, \cdot) \geqq \varrho(l-1, \cdot)$.

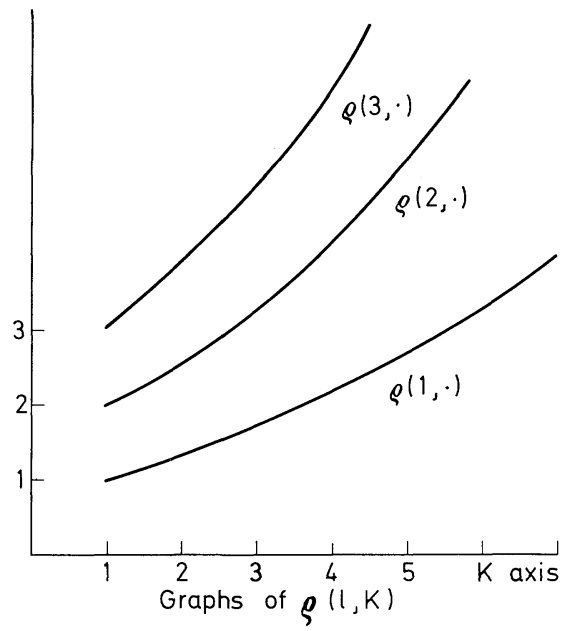

Fig. 1

The purpose of this construction is that for a point $\tilde{n}=\left(n_{1}, n_{2}, \ldots,\right) \in I^{l}$ we have an upper bound, namely $\varrho(l, k)$ on how fast the sequence $\left\{n_{k}\right\}_{k=1}^{\infty}$ can grow.

Definition 3.1. $D_{1}=\left\{\varphi(\tilde{n}) ; \varphi(\tilde{n}) \in L^{2}\left(I^{\infty}, \mu, H_{\tilde{n}}\right), \exists l \ni\|\varphi(\tilde{n})\|_{\tilde{n}}=0\right.$ a.e. for $\left.\tilde{n} \in \tilde{I}^{l}=I^{\infty}-I^{l}\right\}$.

Definition 3.2. $\tau=\left\{\left\{c_{k}\right\}_{k=1}^{\infty} ; c_{k} \in R, \sum_{k=1}^{\infty}\left|c_{k}\right| \varrho(k, k)^{1 / 2}<\infty\right\}$.

We now can state:

Lemma 3.3. $D_{1}$ is dense in $L^{2}\left(I^{\infty}, \mu, H_{\tilde{n}}\right)$ and each $\psi \in D_{1}$ is in the domain of any finite linear combination of $q_{k}$ 's; further, if $\left\{c_{k}\right\}_{k=1}^{\infty} \in \tau$, then 
$\sum_{k=1}^{\infty} c_{k} q_{k} \psi$ converges. Thus, $\sum_{\substack{k=1 \\ \infty}}^{\infty} c_{k} q_{k}$ is a well-defined symmetric operator on $D_{1}$. The same is true of $\sum_{k=1}^{\infty} c_{k} p_{k}$.

Proof. $D_{1}$ is dense since for any $\varphi(\tilde{n}) \in L^{2}\left(I^{\infty}, \mu, H_{\tilde{n}}\right), \int_{\tilde{I}^{l}}\|\varphi(\tilde{n})\|_{\tilde{n}}^{2} d \mu(\tilde{n}) \rightarrow 0$ as $l \rightarrow \infty$. If $\psi \in D_{1}$, then $\psi \in D\left(\left(a_{k} a_{k}^{*}\right)^{1 / 2}\right)$ which implies $\psi \in D\left(q_{k}\right)$. Since the $q_{k}$ commute with each other (i.e. the groups and therefore the spectral projectors commute) $\psi$ is in the domain of any finite linear combination of $q_{k}$. Since $\|\psi(\tilde{n})\|_{\tilde{n}}=0$ a.e. for $\tilde{n} \in \tilde{I}^{l}$ for some $l$,

$$
\begin{aligned}
\left\|\left(a_{k} a_{k}^{*}\right)^{1 / 2} \psi\right\|^{2} & =\int_{I^{\infty}}\left(n_{k}+1\right)\|\psi(\tilde{n})\|_{\tilde{n}}^{2} d \mu(\tilde{n}) \\
& =\int_{I^{l}}\left(n_{k}+1\right)\|\psi(\tilde{n})\|^{2} d \mu(\tilde{n}) \\
& \leqq(\varrho(l, k)+1)\|\psi\|^{2} .
\end{aligned}
$$

Thus for $M, N \geqq l$,

$$
\begin{aligned}
\left\|\sum_{k=M}^{N} c_{k} q_{k} \psi\right\| & \leqq \sum_{k=M}^{N}\left|c_{k}\right|\left\|q_{k} \psi\right\| \leqq \sum_{k=M}^{N}\left|c_{k}\right|\left\|\left(a_{k} a_{k}^{*}\right)^{1 / 2} \psi\right\| \\
& \leqq \sum_{k=M}^{N}\left|c_{k}\right|(\varrho(l, k)+1)^{1 / 2} \\
& \leqq \sum_{k=M}^{N}\left|c_{k}\right|(\varrho(k, k)+1)^{1 / 2} \rightarrow 0
\end{aligned}
$$

as $M, N \rightarrow \infty$. Therefore $\sum c_{k} q_{k}$ is well-defined and symmetric on $D_{1}$ (since it is the strong limit of symmetric operators). The proof for $\sum_{k=1}^{\infty} c_{k} p_{k}$ is the same.

\section{\$ 4. Proof of the Theorem}

The theorem is a result of the following sequence of lemmas. We denote by $\left(a_{k}^{\#}\right)^{m}$ any $m$-fold product of $a_{k}$ and $a_{k}^{*}$.

Lemma 4.1. Let $\psi \in D_{1}$ then

$$
\left\|\prod_{i=1}^{N}\left(a_{k_{\imath}}^{\#}\right)^{m_{i}} \psi\right\|^{2} \leqq\left(\sum_{i=1}^{N} m_{i}\right)^{\sum_{i=1}^{N} m_{i}}\left\|\prod_{i=1}^{N}\left(a_{k_{i}} a_{k_{\imath}}^{*}\right)^{\frac{m_{i}}{2}} \psi\right\|^{2} .
$$

Proof. The proof is a straightforward calculation. Let $s_{i}^{*}$ and $s_{i}$ be respectively the number of $a_{k_{i}}^{*}$ and the number of $a_{k_{i}}$ in the product $\left(a_{k_{i}}^{\#}\right)^{m_{i}}$. Let $\delta_{k_{i}}=s_{i}^{*}-s_{i}$ and let $\delta$ be the sequence of integers which for 
all $i$ has $\delta_{k_{i}}$ in the $k_{i}$ th position and has zeros elsewhere. Then

$$
\begin{aligned}
\left\|\prod_{i=1}^{N}\left(a_{k_{i}}^{\#}\right)^{m_{l}} \psi\right\|^{2} & =\int_{I^{\infty}}\left(\prod_{i=1}^{N} \prod_{j=1}^{m_{i}} \alpha_{j}^{i}(\tilde{n})\right)\|\psi(\tilde{n}-\delta)\|_{\tilde{n}}^{2} d \mu(\tilde{n}-\delta) \\
& =\int_{I^{\infty}}\left(\prod_{i=1}^{N} \prod_{j=1}^{m_{2}} \alpha_{j}^{i}(\tilde{n}+\delta)\right)\|\psi(\tilde{n})\|^{2} d \mu(\tilde{n})
\end{aligned}
$$

where $\alpha_{j}^{i}(\tilde{n})$ is a function on $I^{\infty}$ satisfying $0 \leqq \alpha_{j}^{i}(\tilde{n}) \leqq n_{k_{l}}+1+s_{i}$. Thus,

$$
\alpha_{j}^{i}(\tilde{n}+\delta) \leqq\left(n_{k_{i}}+1+s_{i}+\delta_{k_{2}}\right) \leqq n_{k i}+1+s_{i}^{*} \leqq n_{k_{t}}+1+m_{i},
$$

so,

Therefore,

$$
\prod_{j=1}^{m_{2}} \alpha_{j}^{i}(\tilde{n}+\delta) \leqq m_{i}^{m_{i}}\left(n_{k_{l}}+1\right)^{m_{\imath}}
$$

$$
\begin{aligned}
\left\|\prod_{i=1}^{N}\left(a_{k_{i}}^{\#}\right)^{m_{l}} \psi\right\|^{2} & \leqq \prod_{i=1}^{N} m_{i}^{m_{t}} \int_{I^{\infty}} \prod_{i=1}^{N}\left(n_{k_{i}}+1\right)^{m_{i}}\|\psi(\tilde{n})\|_{\tilde{n}}^{2} d \mu(\tilde{n}) \\
& \leqq\left(\sum_{i=1}^{N} m_{i}\right)^{\left(\sum_{i=1}^{N} m_{t}\right)}\left\|\prod_{i=1}^{N}\left(a_{k_{i}} a_{k_{i}}^{*}\right)^{\frac{m_{i}}{2}} \psi\right\|^{2} .
\end{aligned}
$$

Definition 4.2. We denote by $D$ the set of vectors of the form $\mathscr{P} \psi$, where $\psi \in D_{1}$ and $\mathscr{P}$ is a polynomial of fields $\sum_{k=1}^{\infty} c_{k} q_{k}, \sum_{k=1}^{\infty} d_{k} p_{k} ;\left\{c_{k}\right\}_{k=1}^{\infty}$, $\left\{d_{k}\right\}_{k=1}^{\infty} \in \tau$. Different occurrences of the fields in the polynomial may have different test sequences.

Lemma 4.3. $\sum_{k=1}^{\infty} c_{k} q_{k}$ and $\sum_{k=1}^{\infty} c_{k} p_{k}$ are essentially self-adjoint on $D$.

Proof. Let $\mathscr{P}=\prod_{i=1}^{m}\left(\sum_{k_{i}=1}^{\infty} d_{k_{i}}^{i} q_{k_{\imath}}\right)$. If $\psi \in D_{1}$ then $\|\psi\|_{\tilde{n}}=0$ a.e. for $\tilde{n} \in \tilde{I}^{l}$ for some $l$. Thus

$$
\begin{aligned}
\|\mathscr{P} \psi\| & \leqq \sum_{k_{1}, \ldots, k_{m}}\left(\prod_{i=1}^{m}\left|d_{k_{l}}^{i}\right|\right)\left\|\prod_{i=1}^{m} q_{k_{i}} \psi\right\| \\
& \leqq \sum_{k_{1}, \ldots, k_{m}} \prod_{i=1}^{m}\left|d_{k_{i}}^{i}\right| m^{m / 2} 2^{m / 2}\left\|\prod_{i=1}^{m}\left(a_{k_{k}} a_{k_{i}}^{*}\right)^{1 / 2} \psi\right\|
\end{aligned}
$$

(by Lemma 4.1).

$$
\begin{aligned}
\left\|\prod_{i=1}^{m}\left(a_{k_{i}} a_{k_{i}}^{*}\right)^{1 / 2} \psi\right\|^{2} & =\int_{I^{l}} \prod_{i=1}^{m}\left(\tilde{n}_{k_{t}}+1\right)\|\psi(\tilde{n})\|_{\tilde{n}}^{2} d \mu(\tilde{n}) \\
& \leqq\|\psi\|^{2} \prod_{i=1}^{m}\left(\varrho\left(l, k_{i}\right)+1\right)
\end{aligned}
$$


so we have

$$
\begin{aligned}
\|\mathscr{P} \psi\| & \leqq m^{m / 2} 2^{m / 2}\|\psi\| \sum_{k_{1}, \ldots, k_{m}}^{\infty} \prod_{i=1}^{m}\left|d_{k_{i}}^{i}\right|\left(\varrho\left(l, k_{i}\right)+1\right)^{\frac{1}{2}} \\
& =m^{m / 2} 2^{m / 2}\|\psi\| \prod_{i=1}^{m}\left(\sum_{k_{i}=1}^{\infty}\left|d_{k_{i}}^{i}\right|\left(\varrho\left(l, k_{i}\right)+1\right)^{1 / 2}\right) .
\end{aligned}
$$

Since $\left\{d_{k_{1}}^{i}\right\}_{k_{l}=1}^{\infty} \in \tau$, the right-hand side is finite and $\mathscr{P} \psi \in L^{2}\left(I^{\infty}, \mu, H_{\tilde{n}}\right)$. If we let $\varphi_{N}=\prod_{i=1}^{m} \sum_{k_{i}=1}^{N} d_{k_{i}}^{i} q_{k_{\mathrm{t}}} \psi$, then $\varphi_{N} \in D, \varphi_{N} \rightarrow \mathscr{P} \psi$ and an estimate similar to the one above shows that $\sum_{k=1}^{\infty} c_{k} q_{k}\left(\varphi_{N}-\varphi_{M}\right) \rightarrow 0$ as $N, M \rightarrow \infty$. Thus, $\mathscr{P} \psi$ is in the domain of the closure of $\sum_{k=1}^{\infty} c_{k} q_{k}$. Clearly the same proof works if some of the $\sum_{k_{l}=1}^{\infty} d_{k_{\imath}}^{i} q_{k_{\imath}}$ are replaced by $\sum_{k_{l}=1}^{\infty} d_{k_{i}}^{i} p_{k_{i}}$ or if $\mathscr{P}$ is a finite linear combination of such terms. Since any power of $\sum_{k=1}^{\infty} c_{k} q_{k}$ applied to $\mathscr{P} \psi$ is again a vector of the same form, $\mathscr{P} \psi$ is contained in the domain of any power of the closure of $\sum_{k=1}^{\infty} c_{k} q_{k}$. Finally,

$$
\begin{aligned}
& \left\|\left(\sum_{s=1}^{\infty} c_{s} q_{s}\right)^{v} \mathscr{P} \psi\right\| \\
& \leqq \sum_{s_{1}, \ldots, s_{v}, k_{1}, \ldots, k_{m}}\left(\prod_{i=1}^{v}\left|c_{s_{l}}\right|\right)\left(\prod_{i=1}^{m}\left|d_{k_{i}}^{i}\right|\right)\left\|\prod_{i=1}^{v} q_{s_{i}} \prod_{i=1}^{m} q_{k_{l}} \psi\right\| \\
& \leqq(v+m)^{\frac{1}{2}(v+m)}\left(\sum_{s=1}^{\infty}\left|c_{s}\right|(\varrho(l, s)+1)^{1 / 2}\right)^{v} \prod_{i=1}^{m} \sum_{k_{l}=1}\left|d_{k_{l}}\right|\left(\varrho\left(l, k_{i}\right)+1\right)^{1 / 2}
\end{aligned}
$$

Thus $\sum_{v=0}^{\infty} \frac{t^{v}}{v !}\left\|\left(\sum_{s=1}^{\infty} c_{s} q_{s}\right)^{v} \mathscr{P} \psi\right\|<\infty$ and the same proof holds for more general $\mathscr{P}$. Thus each $\varphi=\mathscr{P} \psi$ in $D$ is an analytic vector for $\sum_{s=1}^{\infty} c_{s} q_{s}$ so by a theorem of Nelson [5], $\sum_{s=1}^{\infty} c_{s} q_{s}$ is essentially self-adjoint on $D$. The same proof works for $\sum_{s=1}^{\infty} c_{s} p_{s}$.

Lemma 4.4. If $\left\{c_{k}\right\}_{k=1}^{\infty} \in \tau, \sum_{k=1}^{\infty} c_{k} q_{k}: D \rightarrow D$ and if $\left\{c_{k}^{n}\right\}_{k=1}^{\infty} \stackrel{\tau}{\rightarrow}\left\{c_{k}\right\}_{k=1}^{\infty}$ then $\sum_{k=1}^{\infty} c_{k}^{n} q_{k}$ converges strongly to $\sum_{k=1}^{\infty} c_{k} q_{k}$ on $D$. The same statements hold for $\sum_{k=1}^{\infty} c_{k} p_{k}$. 
Proof. By its definition $D$ is invariant under $\sum_{k=1}^{\infty} c_{k} q_{k}$ and $\sum_{k=1}^{\infty} c_{k} p_{k}$. Suppose $\left\{c_{k}^{n}\right\}_{k=1}^{\infty} \stackrel{\tau}{\rightarrow}\left\{c_{k}\right\}_{k=1}^{\infty}, \mathscr{P}=\prod_{i=1}^{m}\left(\sum_{k_{i}=1}^{\infty} d_{k_{1}}^{i} q_{k_{l}}\right)$, and $\psi \in D_{1},\|\psi\|_{\tilde{n}}=0$ a.e. for $\tilde{n} \in \tilde{I}^{l}$. Then,

$$
\begin{aligned}
& \left\|\left(\sum_{s=1}^{\infty} c_{s}^{n} q_{s}-\sum_{s=1}^{\infty} c_{s} q_{s}\right) \mathscr{P} \psi \mid\right\| \\
& \left.\quad \leqq \sum_{s, k_{1}, \ldots, k_{m}}\left|c_{s}^{n}-c_{s}\right|\left(\prod_{i=1}^{m} \mid d_{k_{l}}^{i}\right)\right)\left\|q_{s} \prod_{i=1}^{m} q_{k_{\imath}} \psi\right\| \\
& \quad \leqq \sum_{s, k_{1}, \ldots, k_{m}}\left|c_{s}^{n}-c_{s}\right|(\varrho(l, s)+1)^{1 / 2} \prod_{i=1}^{m}\left(\varrho\left(l, k_{i}\right)+1\right)^{1 / 2} \\
& \quad \leqq\left(\prod_{i=1}^{m} \sum_{k_{1}=1}^{\infty}\left|d_{k_{l}}^{i}\right|\left(\varrho\left(l, k_{i}\right)+1\right)^{1 / 2}\right) \sum_{s=1}^{\infty}\left|c_{s}^{n}-c_{s}\right|(\varrho(l, s)+1)^{1 / 2} \rightarrow 0
\end{aligned}
$$

as $n \rightarrow \infty$ since

$$
\sum_{s=1}^{\infty}\left|c_{s}^{n}-c_{s}\right|(\varrho(s, s)+1)^{1 / 2} \rightarrow 0 \quad \text { as } \quad n \rightarrow \infty .
$$

We remark that Lemma 4 and the Trotter-Kato Theorem imply that the maps

$$
\begin{aligned}
& \left\{c_{k}\right\}_{k=1}^{\infty} \rightarrow \exp \left(\text { it } \sum_{k=1}^{\infty} c_{k} q_{k}\right) \\
& \left\{c_{k}\right\}_{k=1}^{\infty} \rightarrow \exp \left(\text { it } \sum_{k=1}^{\infty} c_{k} p_{k}\right)
\end{aligned}
$$

are continuous from $\tau$ to the unitary operators with the strong topology. The spaces

$$
\begin{aligned}
& \mathscr{H}=\left\{\left\{c_{k}\right\}_{k=1}^{\infty} ;\left\|\left\{c_{k}\right\}_{k=1}^{\infty}\right\|^{2}=\sum_{k=1}^{\infty}\left|c_{k}\right|^{2} k^{2} \varrho(k, k)<\infty\right\}, \\
& \mathscr{N}=\left\{\left\{c_{k}\right\}_{k=1}^{\infty} ;\left\|\left\{c_{k}\right\}\right\|_{n}^{2}=\sum_{k=1}^{\infty}\left|c_{k}\right|^{2} k^{n} \varrho(k, k)<\infty \text { for all } n\right\}
\end{aligned}
$$

are respectively Hilbert and Nuclear spaces (with stronger topologies) contained in $\tau$.

\section{§ 5. Consequences for Quantum Field Theory}

Suppose that one has constructed quantum fields $\varphi(f, t), \pi(g, t)$ whose exponentials satisfy

$$
\begin{aligned}
& \exp (i \varphi(f, t)) \exp (i \pi(g, t)) \\
& \quad=\exp \left(\dot{-} i \int f(x) g(x) d x\right) \exp (i \pi(g, t)) \exp (i \varphi(f, t))
\end{aligned}
$$


for $f$ anf $g$ in some test function space $T$. In order to use the structure of the Garding-Wightman representations one must choose an orthonormal basis $\left\{f_{n}\right\}$ of $T$ and define $q_{n}=\varphi\left(f_{n}, t\right), p_{n}=\pi\left(f_{n}, t\right)$. This procedure is presently unpopular and is considered at best inelegant. The difficulties are illustrated by the fact that for different choice of bases one can get different Gårding-Wightman representations. However, the real question is not elegance, but how much information about $\varphi(f, t)$ and $\pi(g, t)$ is lost by passing to a basis, proving theorems about $\left\{\varphi\left(f_{n}, t\right)\right.$, $\left.\pi\left(f_{n}, t\right)\right\}$ and then attempting to recover $\varphi(f, t)$ and $\pi(g, t)$. In our opinion this is very much an open question. To illustrate this we will show how the theorem proved in this paper may be used to find Garding domains for certain basis independent representations.

We consider first the approach of Gelfand and Vilenkin [3]. Let $\mathscr{S}\left(R^{m}\right)$ denote Schwartz space (real) and let $V(\cdot), U(\cdot)$ be continuous maps of $\mathscr{S}\left(R^{m}\right)$ into the unitary operators (strong topology) on some separable Hilbert space $X$ satisfying: $V(f) V(g)=V(f+g), U(f) U(g)$ $=U(f+g)$, and

$$
U(f) V(g)=\exp (i(f, g)) V(f) U(g) .
$$

For each $f, g \in \mathscr{S}\left(R^{m}\right)$ the continuity and linearity conditions imply that $U(t f)$ and $V(t g)$ are continuous one-parameter groups whose generators we denote by $\pi(f)$ and $\varphi(g)$. Let $\left\{f_{k}\right\}_{k=1}^{\infty}$ be an orthonormal basis (in terms of the $L^{2}\left(R^{m}\right)$ inner product) such that each $f \in \mathscr{S}\left(R^{m}\right)$ can be expressed $f=\sum_{k=1}^{\infty} c_{k} f_{k}$ where $\left\{c_{k}\right\}_{k=1}^{\infty} \in S$, the rapidly decreasing sequences, and all such sums are in $\mathscr{S}\left(R^{m}\right)$ (for example, one could use products of Hermite functions). Define $U_{k}(t)=U\left(t f_{k}\right), V_{k}(t)=V\left(t f_{k}\right)$, then $\left\{U_{k}(t), V_{l}(t)\right\}$ is a representation of the Weyl relations $(1.1), q_{k}=\varphi\left(f_{k}\right)$, $p_{k}=\pi\left(g_{k}\right)$. Therefore the conclusions of Theorem 1.1 hold for all $\sum_{k=1}^{\infty} c_{k} q_{k}$, $\sum_{k=1}^{\infty} c_{k} p_{k}$ with $\left\{c_{k}\right\}_{k=1}^{\infty} \in \mathcal{N}$ (see (4.2) for definition of $\mathcal{N}$ ). By the linearity of $\varphi(\cdot)$,

and therefore

$$
\sum_{k=1}^{N} c_{k} q_{k}=\varphi\left(\sum_{k=1}^{N} c_{k} f_{k}\right)
$$

$$
\exp \left(i \sum_{k=1}^{N} c_{k} q_{k}\right)=V\left(\sum_{k=1}^{N} c_{k} f_{k}\right) .
$$

Since $\left\{c_{k}\right\}_{k=1}^{N} \stackrel{\mathscr{L}}{\rightarrow}\left\{c_{k}\right\}_{k=1}^{\infty}$, we have by Theorem 1.1 that $\sum_{k=1}^{N} c_{k} q_{k} \rightarrow \sum_{k=1}^{\infty} c_{k} q_{k}$ strongly on $D$, a domain of essential self-adjointness for all of them. By the Trotter-Kato theorem $\exp \left(i \sum_{k=1}^{N} c_{k} q_{k}\right) \rightarrow \exp \left(i \sum_{k=1}^{\infty} c_{k} q_{k}\right)$. Since the 
topology of $\mathcal{N}$ is stronger than that of $S, \sum_{k=1}^{N} c_{k} f_{k} \mathscr{M} \rightarrow \sum_{k=1}^{\infty} c_{k} f_{k}$, so $V\left(\sum_{k=1}^{N} c_{k} f_{k}\right) \rightarrow V\left(\sum_{k=1}^{\infty} c_{k} f_{k}\right)$, which implies $\sum_{k=1}^{\infty} c_{k} q_{k}=\varphi\left(\sum_{k=1}^{\infty} c_{k} f_{k}\right)$. Let $T=\left\{\sum_{k=1}^{\infty} c_{k} f_{k} ;\left\{c_{k}\right\}_{k=1}^{\infty} \in \mathcal{N}\right\}$ with the natural topology. Therefore, we have proven (by Theorem 1.1)

Theorem 5.1. Let $V(\cdot), U(\cdot)$ be a Gelfand-Vilenkin representation (5.2). Then there exists a nuclear space $T \subset \mathscr{S}\left(R^{m}\right)$ (with stronger topology) such that there is a common dense invariant domain of essential selfadjointness for all the generators of the groups $V(t f), U(t g), f, g \in T$.

It is an attractive conjecture that the basis $\left\{f_{k}\right\}_{k=1}^{\infty}$ can be chosen so that $T=\mathscr{S}\left(R^{m}\right)$.

We now consider the approach of I. Segal [6]. A Weyl system is a map $W(\cdot)$ from a complex Hilbert space $H$ to the unitary operators on a Hilbert space $X$ satisfying:

a) for every $z$ and $z^{\prime}$ in $H$,

$$
W(z) W\left(z^{\prime}\right)=\exp \left[\frac{1}{2} i \mathscr{I} m\left(z, z^{\prime}\right)\right] W\left(z+z^{\prime}\right),
$$

b) for every $z \in H, W(t z)$ is weakly continuous at zero as a function of $t$.

From condition b) it follows that $W(z)=\exp (i \Psi(z))$, where $\Psi(z)$ is a self-adjoint operator on $X$ and that the map $z \rightarrow W(z)$ is continuous from finite dimensional subsets of $H$ to the unitary operators with the strong topology. It does not follow that $z \rightarrow W(z)$ is globally continuous, i.e. continuous from $H$ to the unitary operators on $X$. Let $\{z\}_{k=1}^{\infty}$ be an orthonormal basis for $H$ and define

$$
V_{k}(t)=W\left(t z_{k}\right), \quad U_{k}(t)=W\left(t i z_{k}\right) .
$$

Then $\left\{U_{k}(t), V_{k}(t)\right\}$ is a Gårding-Wightman representation, $q_{k}=\Psi\left(z_{k}\right)$, $p_{k}=\Psi\left(i z_{k}\right)$. From Theorem 1.1: there exists a Hilbert space $\mathscr{H}$ (see (4.1) for definition) of sequences so that if $\left\{c_{k}\right\}_{k=1}^{\infty} \in \mathscr{H}, \sum_{k=1}^{\infty} c_{k} q_{k}, \sum_{k=1}^{\infty} c_{k} p_{k}$ make sense on $X$. Let $T=\left\{\sum_{k=1}^{\infty} \alpha_{k} z_{k} ; \alpha_{k} \in \mathbb{C}, \sum\left|\alpha_{k}\right|^{2} k^{2} \varrho(k, k)<\infty\right\}$ with the natural inner product and define for $z=\sum_{k=1}^{\infty} \alpha_{k} z_{k} \in T$,

$$
\begin{aligned}
W^{\prime}(z)= & \exp \left[\frac{1}{2} i \sum_{k=1}^{\infty}\left(\mathscr{R} e\left(\alpha_{k}\right)\right) \mathscr{I} m\left(\alpha_{k}\right)\right] \exp \left[\sum_{k=1}^{\infty} \mathscr{R} e\left(\alpha_{k}\right) q_{k}\right] \\
& \cdot \exp \left[\sum_{k=1}^{\infty} \mathscr{I} m\left(\alpha_{k}\right) p_{k}\right] .
\end{aligned}
$$


$W^{\prime}(z)$ is a globally continuous Weyl system over $T$ which agrees with $W(z)$ on finite linear combinations of the basis elements $z_{k}$. The open question is, to what extent do $W(z)$ and $W^{\prime}(z)$ differ for $z \in T$. Chaiken [1] has shown that if the operators $\left\{W\left(\sum_{k=1}^{N} \alpha_{k} z_{k}\right), N\right.$ arb. $\}$ are irreducible, then $W(z)=\exp (i \lambda(z)) W^{\prime}(z)$ where $\lambda$ is an everywhere defined (in general discontinuous) linear functional on $T$. In this case the generators of $W(z)$ differ from the generators of $W^{\prime}(z)$ by constant multiples of the identity which affect none of the conditions of Theorem 1.1 except the continuity statement 3 ). We therefore have

Theorem 5.2. Let $(W(\cdot), H)$ be a Weyl system on $X$ such that for some orthonormal basis $\left\{z_{k}\right\}_{k=1}^{\infty},\left\{W\left(\sum_{k=1}^{N} c_{k} z_{k}\right)\right\}$ is irreducible. Then there exists a sub-Hilbert space $T \subset H$ such that

a) there is a common dense invariant domain of essential self-adjointness for all the generators of $W(t z), z \in T$.

b) W(z) differs from a globally continuous Weyl system over $T$ by a complex (possibly discontinuous) character of $T$.

B. Simon has pointed out that since the fields which generate $W(z)$ and $W^{\prime}(z)$ differ by a constant multiple of the identity, their truncated vacuum expectation values will be the same. Therefore both systems will have the same scattering theory.

Acknowledgment. The author would like to thank E. Nelson, J.-P. Antoine and especially B. Simon for several stimulating discussions concerning the material in this paper.

\title{
References
}

1. Chaiken, J.: Finite-particle representations and states of the canonical commutation relations. Ann. Phys. 42, $23-80$ (1967).

2. Cook, J.: The mathematics of second quantization. Trans. Am. Math. Soc. 74, 222-245 (1953).

3. Gelfand, I., et N. Vilenkin: Les distributions IV. Paris: Dunod 1967.

4. Garding, L., and A. Wightman: Representations of the commutation relations. Proc. Natl. Acad. Sci. U.S. 40, 622-626 (1954).

5. Nelson, E.: Analytic vectors. Ann. Math. 70, 572-615 (1959).

6. Segal, I.: Mathematical problems of relativistic physics. Providence, R. I.: Am. Math. Soc. 1963.

\author{
M. C. Reed \\ Department of Mathematics \\ Princeton University \\ Princeton, N. J., USA
}

